Original Paper

\title{
Gut Taste Stimulants Alter Brain Activity in Areas Related to Working Memory: a Pilot Study
}

\author{
Anne Christin Meyer-Gerspach ${ }^{\mathrm{a}} \quad$ Claudia Suenderhauf $^{\mathrm{b}} \quad$ Lukas Bereiter $^{\mathrm{a}}$ \\ Davide Zanchi ${ }^{b}$ Christoph Beglinger ${ }^{a}$ Stefan Borgwardt ${ }^{b}$ \\ Bettina K. Wölnerhanssen ${ }^{a}$ \\ ${ }^{a}$ Department of Biomedicine, Division of Gastroenterology, University Hospital Basel, bepartment of \\ Psychiatry, University Hospital Basel, Basel, Switzerland
}

\section{Key Words}

Gut taste receptors $•$ Working memory $•$ Functional MRI $•$ Quinine $\cdot$ Monosodium glutamate - Bitter taste $\cdot$ Umami taste

\begin{abstract}
Background/Aims: Taste perception is one of the most important primary oral reinforcers, driving nutrient and energy intake as well as toxin avoidance. Taste receptors in the gastrointestinal tract might as well impact appetitive or aversive behavior and thus influence learning tasks and a close relation of neural taste processing and working memory networks seems plausible. Methods: In the present pilot study, we determined the effects of five taste qualities "bitter" (quinine), "sweet" (glucose), "sour" (citric acid), "salty" ( $\mathrm{NaCl}$ ) and "umami" (monosodium glutamate, MSG) on working memory processing using functional MRI and their effect on plasma insulin and glucose levels. On six separate occasions, subjects received one of the following test substances dissolved in $200 \mathrm{~mL}$ tap water via a nasogastric tube (to circumvent the oral cavity): 1) $2 \mathrm{~g}$ citric acid corresponding to $52 \mathrm{mM}, 2) 2 \mathrm{~g} \mathrm{NaCl} ; 171 \mathrm{mM}, 3$ ) $0.017 \mathrm{~g}$ quinine; $0.26 \mathrm{mM}$, 4) $1 \mathrm{~g}$ monosodium glutamate; $30 \mathrm{mM}$, 5) $25 \mathrm{~g}$ glucose; $694 \mathrm{mM}$ and 6) $200 \mathrm{~mL}$ tap water (placebo). Results: The taste qualities "bitter" and "umami" significantly altered brain activation patterns in the primary gustatory cortex as well as in subcortical structures, previously reported to be involved in emotional learning and memory. In contrast, glucose did not reveal any statistically significant brain activation difference. Working memory performance was not different over the six treatments. Plasma insulin and glucose levels were not affected by the different taste substances (MSG, quinine, $\mathrm{NaCl}$ and citric acid). Conclusions: in this pilot trial, we demonstrate that acute intragastric administration of different taste substances does not affect working memory performance in humans. However, "umami" and "bitter" have effects on brain areas involved in neural working memory, overpowering the effects of "sweet", "salty" and "sour" reception.

A. C. Meyer-Gerspach, and C. Suenderhauf contributed equally to this work.

\begin{tabular}{ll}
\hline Bettina K. Wölnerhanssen, M.D. & Department of Biomedicine, University Hospital Basel \\
& CH-4031 Basel, (Switzerland) \\
& Tel. +41 613287378, E-Mail bettina.woelnerhanssen@usb.ch
\end{tabular}


Meyer-Gerspach et al.: Gut Tastes Modify Brain Activity During a Working Memory Task

\section{Introduction}

The sensation of the five fundamental tastes sweet, sour, salty, umami (taste quality described as "delicious" or "savory") and bitter is strongly conserved across species, in line with the omnipresent drive to ingest nutrient-rich food (sweet, umami) and to avoid toxic substances (bitter) or strong acids (sour) that are present in spoilt foods and unripe fruits $[1,2]$. Although there is a certain innate disposition to like or dislike certain tastes, these preferences can be unlearned or overwritten. Especially bitter tasting substances can be learned to be perceived as pleasant, arguably due to the presence of some pharmacological effect; moreover, it might be an adaptive advantage to recognize them as beneficial.

Taste receptors are not only functionally expressed on taste buds of the tongue but also in other organs such as the gastrointestinal (GI) tract (Fig. 1), pancreas, and the brain [1-4]. Intestinal tasting might be involved in learning tasks, involving detection of caloric value or of toxic effects of food components, ultimately enabling their recognition and initiation of appetitive or aversive behavior.

Functional brain MRI is a rather novel method to investigate effects of different ingested nutrients on regional brain activation and is a helpful tool to examine the gut brain axis. In a recent fMRI study, we have demonstrated that intragastrically administered glucose and fructose have dissociable effects on resting state brain activity in limbic networks [5]. The exact interplay of intestinal taste receptor stimulation and brain activity in humans is not yet fully understood. Given the close relationship of food foraging related learning and taste perception and recognition, we hypothesized that different taste qualities might modulate brain activity during working memory processing. Our primary goal was to examine whether intestinal administration of physiological concentrations of different taste stimuli sensed by taste receptors located in the upper gastrointestinal tract leads to signals that are conducted to and processed by the central nervous system and would evoke different blood-oxygendependent (BOLD) response during working memory processing. While studies on rats have been carried out and demonstrated activation of forebrain regions in functional MRI in response to intragastric infusions of taste solutions $[6,7]$ no previous trials - to the best of our knowledge - examined human gastrointestinal taste receptor stimulation and afferences to the central nervous system by functional brain MRI.

\section{Materials and Methods}

Clinical trial registry

ClinicalTrials No. NCT02255812, https://clinicaltrials.gov/

Study approval

The protocol was approved by the Ethics Committee of Basel, Switzerland (EKBB: 298/12) and conducted in accordance with the principles of the Helsinki Declaration of 1975 as revised in 1983. Subjects were recruited by word of mouth over a period of eight months (01. March 2013 - 11. October 2013). Each participant gave written informed consent prior to inclusion in the study. The Clinical Trial Registration Number is: NCT02255812.

\section{Subjects}

Twelve right-handed male volunteers (mean age: $24.1 \pm 1.2$ years, range: $19-34$ years and mean BMI: $22.8 \pm 0.5 \mathrm{~kg} / \mathrm{m}^{2}$ (range: $19.3-24.822 .9 \mathrm{~kg} / \mathrm{m}^{2}$ ) were analyzed. Only right-handed males were included, as previous studies indicated lateralization in the gustatory cortex according to handedness [8]. Each participant underwent a medical interview and a laboratory screening. Exclusion criteria were: smoking, substance abuse, regular intake of medications, medical or psychiatric illness, fructose intolerance and any contraindication to MRI (e.g. claustrophobia, non-removable metal devices), and any abnormalities detected upon laboratory screening. Of the twelve subjects originally recruited two had to be excluded as they did not 


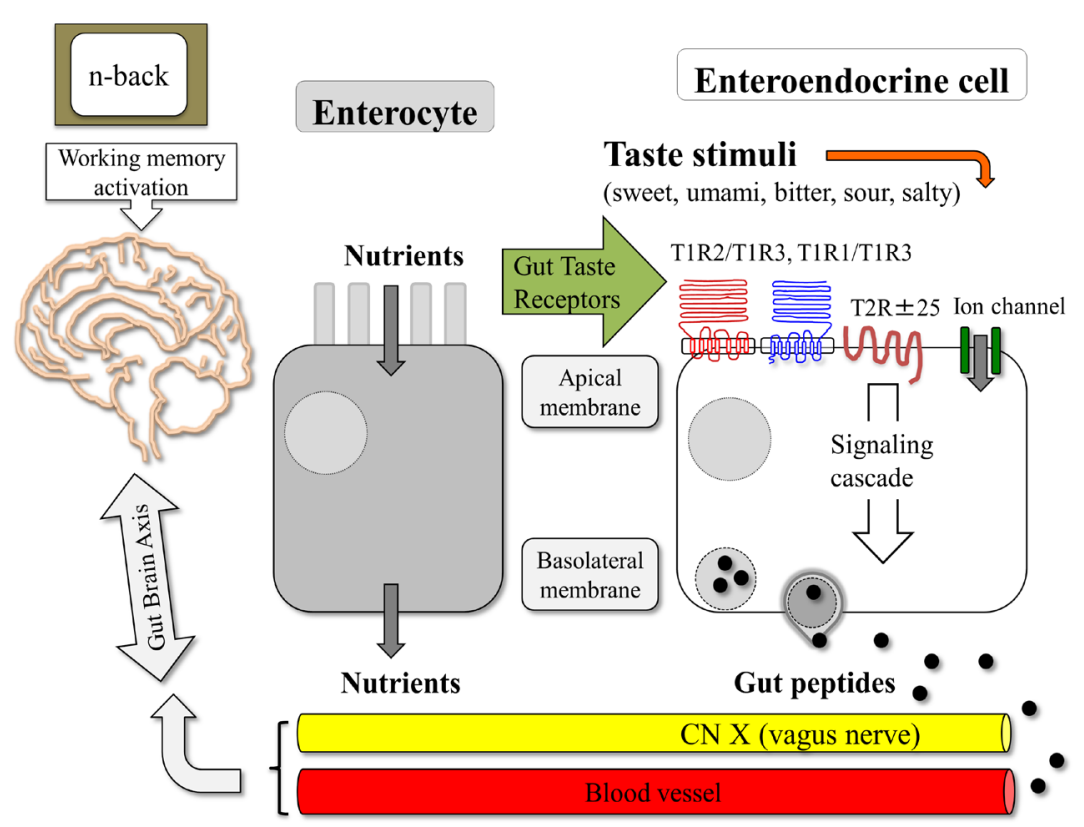

Fig. 1. Intestinal taste perception and gut brain axis. The majority of cells lining the intestinal lumen are common enterocytes their main purpose being the digestion and resorption of nutrients. Apart from these, specialized cells - the so-called enteroendocrine cells - are found throughout the intestinal tract. They act as chemosensors and various receptors are expressed on the luminal (apical) surface. Among these, taste receptors are found for sweet (T1R2/T1R3), umami (T1R1/T1R3) and bitter T2R \pm 25 as well as ion channels for salty $(\mathrm{Na}+)$ and sour $(\mathrm{H}+)$ ions. Signals are transferred to the central nervous system (gut brain axis) by means of peptides which act directly on the vagal nerve and are also released into the blood and thus reach the central nervous system via the blood stream.

meet the eligibility criteria. One drop-out had to be replaced. Hence, complete data from 10 subjects were obtained for analysis. The purpose of this study was to gain basic information on the physiologic role of the aforementioned taste stimuli on cerebral BOLD response. Samples size of this purely observational study was chosen on the basis of practical considerations rather than statistical estimation.

\section{Study design and experimental procedures}

The study was a randomized (balanced), placebo-controlled, double-blind, cross-over trial and was carried out at the Phase one Research Unit of the University Hospital of Basel. Each subject was administered citric acid, $\mathrm{NaCl}$, quinine, monosodium glutamate, glucose and placebo on six separate occasions. The treatment order was randomized within a subject and the time between these visits was at least 3 days.

After an overnight fast of at least 10 hours, an $8 \mathrm{~F}$ polyvinyl nasogastric tube was inserted into the stomach through an anaesthetized nostril and its intragastric position was confirmed by rapid injection of $10 \mathrm{~mL}$ of air and auscultation of the upper abdomen. An antecubital vein catheter was inserted into a forearm vein for blood collection. After taking two fasting blood samples ( $t=-10$ and $-1 \mathrm{~min}$ ), subjects received one of the following test substances dissolved in $200 \mathrm{~mL}$ tap water at room temperature:

1) $2 \mathrm{~g}$ citric acid corresponding to $52 \mathrm{mM}$,

2) $2 \mathrm{~g} \mathrm{NaCl} ; 171 \mathrm{mM}$,

3) $0.017 \mathrm{~g}$ quinine; $0.26 \mathrm{mM}$,

4) $1 \mathrm{~g}$ monosodium glutamate; $30 \mathrm{mM}$,

5) $25 \mathrm{~g}$ glucose; $694 \mathrm{mM}$ and

6) $200 \mathrm{~mL}$ tap water (placebo).

Glucose monohydrate was purchased from Haenseler AG (Herisau, Switzerland), citric acid, quinine and monosodium glutamate were purchased from Sigma-Aldrich (Schnelldorf, Germany) and $\mathrm{NaCl}$ from Schweizer Salinen AG (Schweizerhalle, Switzerland). In order to maintain the blind, differing persons prepared and administered the treatment. Concentrations of test substances were selected to represent physiologically consumed amounts (e.g. bitter: like in tonic water, sour: like in a glass of orange juice). 
Meyer-Gerspach et al.: Gut Tastes Modify Brain Activity During a Working Memory Task

Solutions were freshly prepared at each occasion and the subjects received the solutions via nasogastric tube over 2 minutes ( $\mathrm{t}=0-2 \mathrm{~min}$ ) while sitting in the MR room. The person preparing the solution and the person administering the treatment were not identical, so that deliverer and recipient were blinded. After administration, the feeding tube was removed and within 5 minutes after administration of the test solution the subjects underwent MRI scanning. The participant's vital signs (blood pressure, heart rate) were monitored before and after each study day.

Blood sampling and laboratory analysis

At regular time intervals (15, 30, 45 and 60 min after administration of the test solution), additional blood samples were taken. The blood samples were collected on ice into tubes containing EDTA ( $6 \mu \mathrm{mol} / \mathrm{L})$, a protease inhibitor cocktail (Complete ${ }^{\circledR}$, EDTA-free, 1 tablet/50 mL blood; Roche, Mannheim, Germany) and a dipeptidylpeptidase IV inhibitor $(10 \mu \mathrm{L} / \mathrm{mL}$; Millipore Corporation, St. Charles, Missouri, USA). Tubes were centrifuged at $4 \mathrm{C}$ at $3000 \mathrm{rpm}$ for $10 \mathrm{~min}$ and plasma samples were processed into different aliquots. All samples were stored at $-70 \mathrm{C}$ until analysis of plasma insulin and glucose.

Plasma Insulin was measured with a commercially available ELISA kit (Abnova, Taipei City, Taiwan). The intra- and inter-assay coefficients of variation are below $8.1 \%$ and $8.5 \%$. Plasma glucose concentration was measured by a glucoseoxidase method (Rothen Medizinische Laboratorien AG, Basel, Switzerland). The intra- and inter-assay coefficient of variation is below $2.9 \%$ and $3.9 \%$, respectively.

\section{fMRI paradigm}

The functional imaging protocol consisted of a block-designed n-back to assess working memory related processing, which has been previously used in pharmacological fMRI studies $[9,10]$. During the task, a sequence of letters was presented to the subjects, with one second presentation time and two seconds inter-stimulus time. The n-back task required the participants to indicate whether the presented letter matched the one from n-steps earlier and $n$ was set to 1 and 2 in the present study. In addition, the paradigm contained a baseline condition ( 0 -back task), where subjects had to press a button whenever the letter "X" was presented. The button box was placed in the subjects' right hand. Sequences of the three conditions were repeatedly presented in pseudo-randomized order $(2 \times 1$-back, $3 \times 2$-back and $5 \times 0$-back) to cancel out any onset time-related bias in activation patterns. The n-back paradigm used in the present study had a total duration of 5 min and 20 seconds.

\section{MRI Image acquisition}

Images were obtained on a 3T scanner (Siemens Magnetom Verio) with a 12-channel radio-frequency phased-array head coil. Whole-brain functional imaging was performed 18 minutes post-treatment using a gradient echo planar sequence. An interleaved descending scan was performed with the following parameters: $\mathrm{TR}=2500 \mathrm{~ms}, \mathrm{TE}=28 \mathrm{~ms}$, flip angle $=82^{\circ}$, field of view $=228 \times 228 \mathrm{~mm}^{2}, 38$ slices, slice thickness: $3 \mathrm{~mm}$; voxel size $=3 \times 3 \times 3 \mathrm{~mm}^{3}$. In total, $126 \mathrm{EPI}$ volumes and a high-resolution T1-weighted magnetization prepared rapid acquisition gradient echo (MPRAGE) image was acquired (TR = $2000 \mathrm{~ms}$; TE $=3.37 \mathrm{~ms}$; flip angle $=8^{\circ}$; inversion time $=1000 \mathrm{~ms} ; 176$ slices; slice thickness $=1 \mathrm{~mm}$; voxel size $=1 \mathrm{x} 1 \mathrm{x}$ $\left.1 \mathrm{~mm}^{3}\right)$.

\section{Analysis of fMRI N-Back data}

Analysis was performed using statistical parametric mapping software SPM12 (http://www.fil.ion. ucl.ac.uk/spm/) on Matlab (MATLAB and Statistics Toolbox Release 2015a, The MathWorks, Inc., Natick, Massachusetts, United States) [11]. Functional volumes were slice time corrected (interleaved descending), spatially realigned to correct for head motion and co-registered to corresponding structural volumes, which were realigned and transferred to standard stereotactic MNI space (Montreal Neurological Institute) by using DARTEL normalization [12]. We used a $6 \mathrm{~mm}$ full width at half maximum Gaussian kernel to smooth the BOLD volumes.

Prior to model estimation, the time series blood oxygen level-dependent (BOLD) data was temporally filtered (high pass) and spatially smoothed in an effort to improve the signal-to-noise ratio. We applied a general linear model on subject level. The design matrix included four conditions (with onset times of 0-back, 1-back, 2-back in seconds) in a block design. Movement was accounted by introducing nuisance 
Meyer-Gerspach et al.: Gut Tastes Modify Brain Activity During a Working Memory Task

regressors for 6 spatial axes. Onset times of each test condition for each subject were convolved with a canonical hemodynamic response function (autoregressive AR (0) model). Low frequency noise was removed by a high-pass filter (high-pass filter cutoff of 128s). On first level analysis, we focused our analysis on 2-back versus 0-back contrasts to assess the highest possible working memory load during the task and to discriminate working memory from attention tasks $[13,14]$.

On group (second) level, we compared all treatments to each other using an ANOVA within-subject design. Only contrasts which were statistically significant were reported. The high-threshold significance level for activation was set to $p<0.001$ peak level and $p<0.05$ cluster level. Here we report cluster level $p$ values. We did not correct for multiple comparisons due to moderate effect expected on a small sample size, typical of the present pilot study design. P values reported refer to significance at cluster level. Degrees of freedom were 45. In addition, n-back performance (percent correct answers) and reaction times (ms) of all subjects were compared over all visits and over all treatments by means of repeated measures one-way ANOVA (GraphPad Prism Version 6, GraphPad Software, San Diego, USA).

\section{Statistics}

Descriptive statistics were used for demographic variables such as age, weight, height, and BMI. Plasma insulin and glucose profiles were analyzed by calculating area under the concentration-time curve (AUC 0-60) from baseline values. The parameters were tested for normality by the Shapiro-Wilk test method. Repeated measures ANOVA and simple contrasts with Bonferroni correction were used to test for significant differences between the different taste substances. All statistical analysis was done using the statistical software package SPSS for Windows Version 19.0 (SPSS Inc., Chicago, USA). Values were reported as mean \pm SEM. Differences were considered to be significant with $p \leq 0.05$.

\section{Results}

Complete data sets from 10 subjects were obtained and analyzed. There were no adverse events observed throughout the whole study duration.

\section{$N$-Back performance}

The mean performance over all trials and subjects (measured as percent correct answers) was $70 \%$ correct answers. There was no significant difference in n-back performance between the six different visits. Moreover, there was no difference of working memory performance across different treatments. Mean reaction time was $817.9+/-65.3$ $\mathrm{ms}$. When we compared reaction times over different treatments and different visits, we did not find any statistically significant difference. Figures of n-back performance and reaction times are given in supplemental material.

\section{Effects of taste receptors on neural response during working memory processing}

We found significant clusters contrasting the treatments of quinine versus $\mathrm{NaCl}$ (cluster 1 , precentral gyrus, $p \leq 0.001, \mathrm{z}=5.18$; cluster 2 , medial frontal gyrus, $p=0.001, \mathrm{z}=4.20$ ) (upper panel, Fig. 2) and glucose (cluster 1, medial frontal gyrus, $p=0.033, \mathrm{z}=4.47$; cluster 2. Lingual and calcarine gyrus, $p=0.005 ; \mathrm{z}=4.42$, cluster 3 , precentral gyrus, $p=0.037$, $\mathrm{z}=4.24$ ) (lower panel, Fig. 2). Quinine treatment led mainly to recruitment of additional clusters in the right precentral and medial frontal areas when compared to saline condition. When compared to glucose, we observed right hemispherical activation in the lingual and calcarine gyrus, pre- and postcentral areas as well as medial frontal areas.

Monosodium glutamate (MSG) treatment recruited significantly more clusters compared to saline (Fig. 3). For the contrast of MSG versus $\mathrm{NaCl}$, these areas were located in the gyrus precentralis bilaterally (cluster 1 , left hemisphere, $p \leq 0.001, \mathrm{z}=4.71$; cluster 2 , right hemisphere, $p=0.004, \mathrm{z}=4.54$ ), in the left gyrus postcentralis (cluster $3, p=0.002, \mathrm{z}$ $=4.42$ ) and the left precuneus (cluster $4, p=0.036, \mathrm{z}=4.07$ ), the left insular lobe (cluster 5 , $p=0.006, \mathrm{z}=3.89$ ) and the right cingulate gyrus (cluster $6, p=0.017, \mathrm{z}=3.87$ ). Table 1 lists statistically significant contrasts and anatomical mapping of activated clusters. 


\section{SIGUNAis}

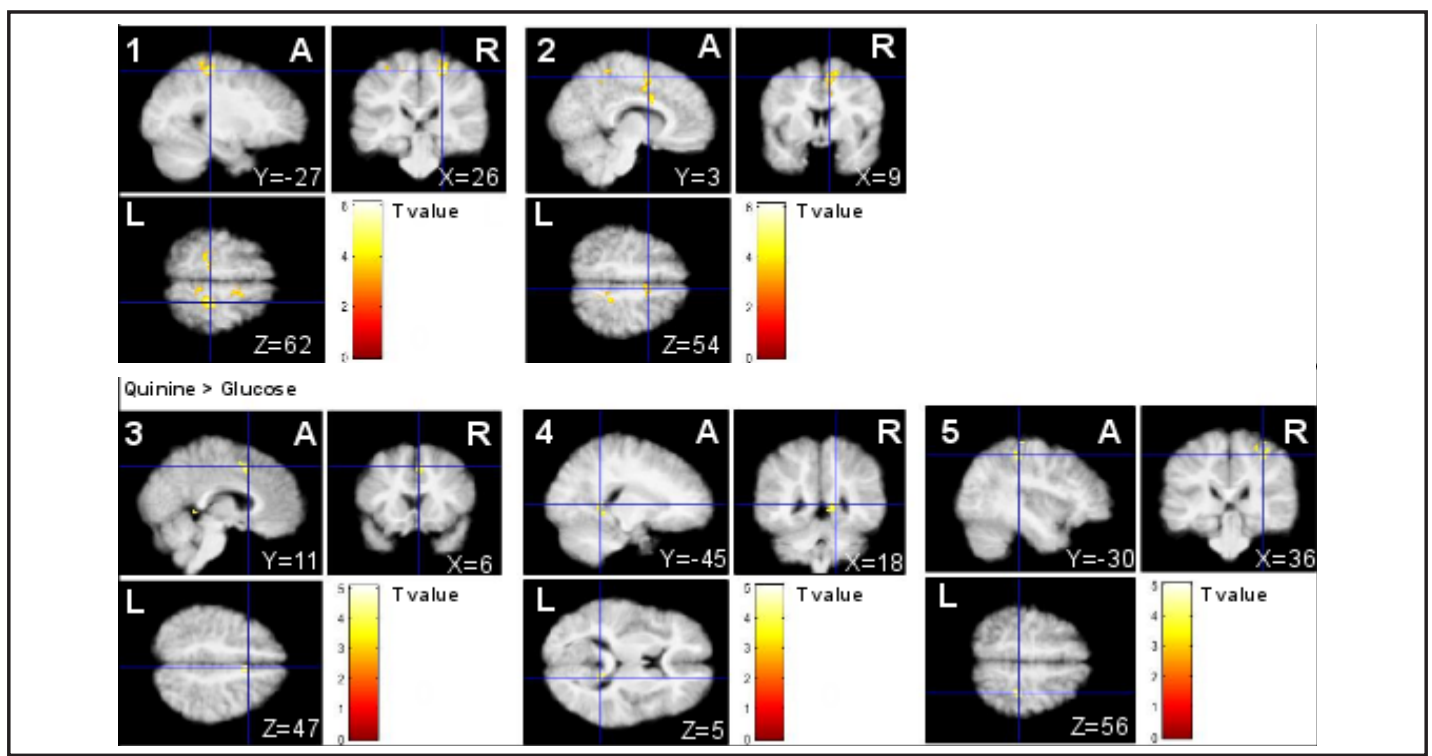

Fig. 2. Contrasts of quinine. A and B show significant activity in the right hemispheric frontal lobe during working memory processing (2-back $>0$-back) under quinine compared to $\mathrm{NaCl}$ treatment. Clusters are located in precentral (A) (MNI coordinates $x, y, z=26,-27,62 ; p \leq 0.001$ ) and medial frontal gyrus (B) (MNI coordinates $x, y, z=9,3$, $54 ; \mathrm{p}=0.001)$. $\mathrm{C}, \mathrm{D}$ and $\mathrm{E}$ show the three significantly increased activated clusters located in the right hemisphere in the contrast of quinine compared to glucose treatment. Activation is shown in the right hemispheric medial frontal gyrus (MNI coordinates $\mathrm{x}, \mathrm{y}, \mathrm{z}=6,11,47 ; \mathrm{p}=0.033$ ), right gyrus calcarinus (MNI coordinates $\mathrm{x}, \mathrm{y}, \mathrm{z}=18,-45,5 ; \mathrm{p}$ $=0.005$ ) and in the right precentral gyrus (MNI coordinates $x, y, z=36,30,56 ; p=0.037$ ), respectively. We used a significance high threshold of $\mathrm{p}=0.001$ and did not correct for multiple comparisons.

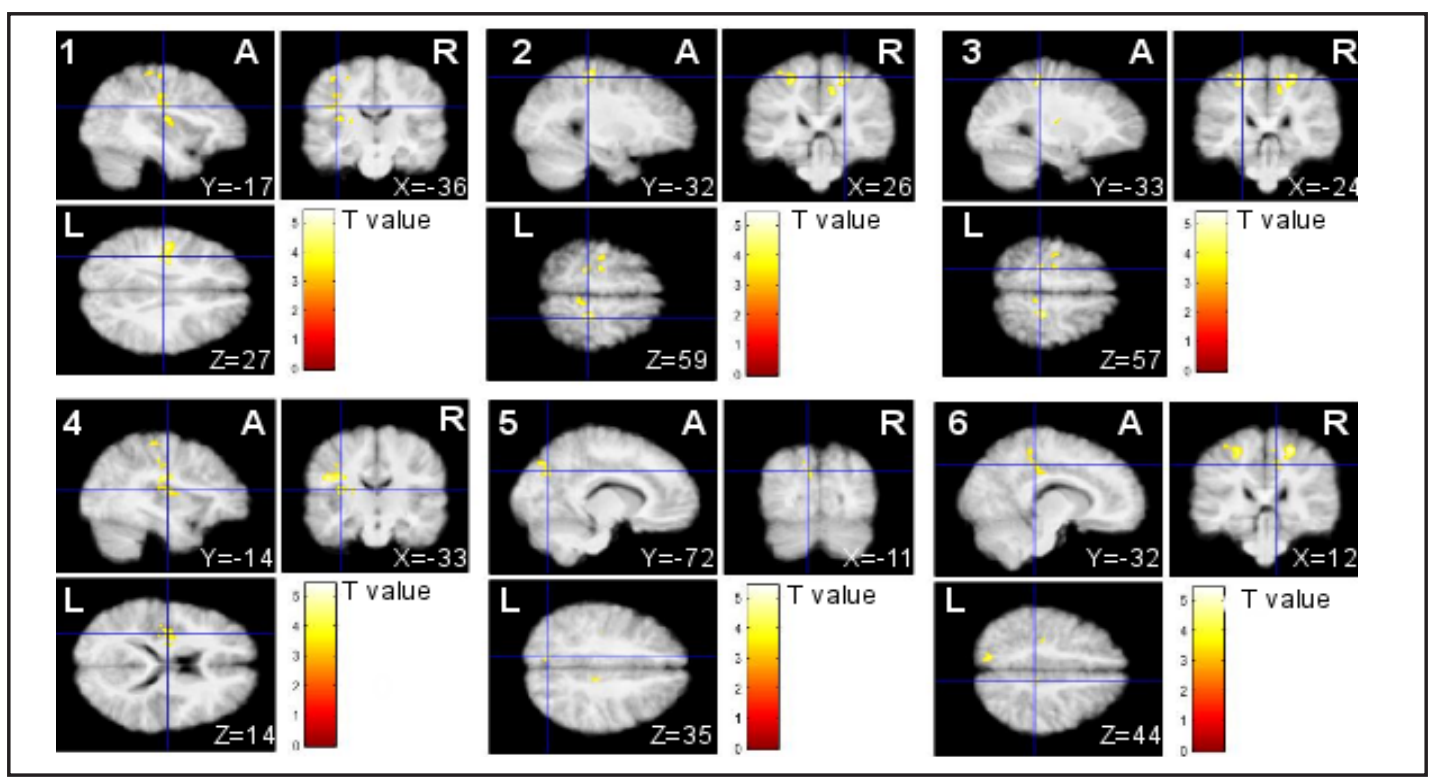

Fig. 3. Contrasts of MSG. Figure 3 shows the six significantly activated clusters in working memory processing (2-back > 0-back) under intragastric MSG compared to $\mathrm{NaCl}$ treatment. Each panel depicts the slice where the corresponding cluster is optimally illustrated. A and B focus on the two clusters in the left and right hemispheric precentral gyrus (MNI coordinates $x, y, z=-36,-17,27 ; p \leq 0.001$; MNI coordinates $x, y, z=26,-32,59 ; p=0.004$ ), respectively. Significantly activated clusters in the left postcentral gyrus (C, MNI coordinates $x, y, z=-24,-33,57 ; p$ $=0.002)$ and left hemispheric operculum (D, MNI coordinates $\mathrm{x}, \mathrm{y}, \mathrm{z}=-33,-14,14 ; \mathrm{p}=0.006$ ) are shown in $\mathrm{C}$ and D. E and F depict left hemispheric activation in the precuneus (MNI coordinates $x, y, z=-11,-72,35 ; p=0.036$ ) and the cingulate gyrus (MNI coordinates $\mathrm{x}, \mathrm{y}, \mathrm{z}=12,-32,44 ; \mathrm{p}=0.017$ ). We used a significance high threshold of $\mathrm{p}=0.001$ and did not correct for multiple comparisons. 
Table 1. Anatomical mapping and MNI coordinates of significantly activated clusters ( $\mathrm{p}$ - values associated with significant clusters) during the n-back task are shown. Indicated brain areas were significantly more activated under a working memory task when quinine was administrated compared to $\mathrm{NaCl}$ and glucose. Intragastrically administered monosodium glutamate (MSG) showed significant cluster activation compared to $\mathrm{NaCl}$ solution. Peak level threshold of $\mathrm{p}<0.001$ and a cluster level threshold of $\mathrm{p}<0.05$ were used. We report here cluster level $\mathrm{p}$ values. Cluster size is given as voxels. 1Brodmann areas

\begin{tabular}{|c|c|c|c|c|c|c|c|}
\hline Contrast & $\begin{array}{c}\text { Cluster } \\
\text { No }\end{array}$ & $\begin{array}{c}\text { MNI } \\
\text { Coordinates }\end{array}$ & $\begin{array}{l}\text { Significance at } \\
\text { cluster level }(p)\end{array}$ & $\begin{array}{c}\text { Cluster } \\
\text { size } \\
\text { (voxels) }\end{array}$ & $\begin{array}{c}Z \text { scores } \\
\text { (at peak } \\
\text { level) }\end{array}$ & Hemisphere & Anatomical area \\
\hline \multirow[t]{3}{*}{ Quinine > Glucose } & 1 & $6,11,47$ & 0.033 & 226 & 4.47 & right & $\begin{array}{l}\text { Frontal lobe, medial frontal } \\
\text { gyrus, supplementary motor area }\end{array}$ \\
\hline & 2 & $18,-45,5$ & 0.005 & 326 & 4.42 & right & Lingual gyrus, calcarine gyrus \\
\hline & 3 & $36,-30,56$ & 0.037 & 220 & 4.24 & right & $\begin{array}{l}\text { Frontal lobe, precentral gyrus, } \\
\mathrm{BA}^{1} 4 \text {, postcentral gyrus }\end{array}$ \\
\hline \multirow[t]{2}{*}{ Quinine $>\mathrm{NaCl}$} & 1 & $26,-27,62$ & $<0.001$ & 1011 & 5.18 & right & $\begin{array}{l}\text { Frontal lobe, precentral gyrus, } \\
\mathrm{BA}^{1} 4,3,2,5 \text {, and } 7\end{array}$ \\
\hline & 2 & $9,3,54$ & 0.001 & 451 & 4.20 & right & $\begin{array}{l}\text { Frontal lobe, medial frontal } \\
\text { gyrus, } \mathrm{BA}^{1} 6\end{array}$ \\
\hline \multirow[t]{6}{*}{$\mathrm{MSG}>\mathrm{NaCl}$} & 1 & $-36,-17,27$ & $<0.001$ & 853 & 4.71 & left & $\begin{array}{l}\text { Frontal lobe, precentral gyrus, } \\
\mathrm{BA}^{1} 3,4 \text {, and } 44\end{array}$ \\
\hline & 2 & $26,-32,59$ & 0.004 & 340 & 4.54 & right & $\begin{array}{l}\text { Frontal lobe, precentral gyrus, } \\
\mathrm{BA}^{1} 3 \mathrm{~b} \text { and } 4\end{array}$ \\
\hline & 3 & $-24,-33,57$ & 0.002 & 392 & 4.42 & left & $\begin{array}{l}\text { Parietal lobe, postcentral gyrus, } \\
\mathrm{BA}^{1} 3\end{array}$ \\
\hline & 4 & $-33,-14,14$ & 0.006 & 320 & 4.07 & left & $\begin{array}{l}\text { Insular lobe, opercular areas } O P \\
1,2 \text {, and } 3\end{array}$ \\
\hline & 5 & $-11,-72,35$ & 0.036 & 222 & 3.89 & left & Parietal lobe, precuneus, $\mathrm{BA}^{1} 7$ \\
\hline & 6 & $12,-32,44$ & 0.017 & 261 & 3.87 & right & Cingulate gyrus, $\mathrm{BA}^{1} 5$ \\
\hline
\end{tabular}

Fig. 4. Plasma insulin and glucose levels. Plasma insulin and glucose concentrations in response to intragastric loads of different taste substances. Data are expressed as mean \pm SEM. Repeated measures ANOVA and simple contrasts with Bonferroni correction were used to test for significant differences between the different taste substances. ${ }^{*}, \mathrm{p} \leq 0.05$, statistically significant difference vs tap water and the other taste substances. $\mathrm{N}=10$.

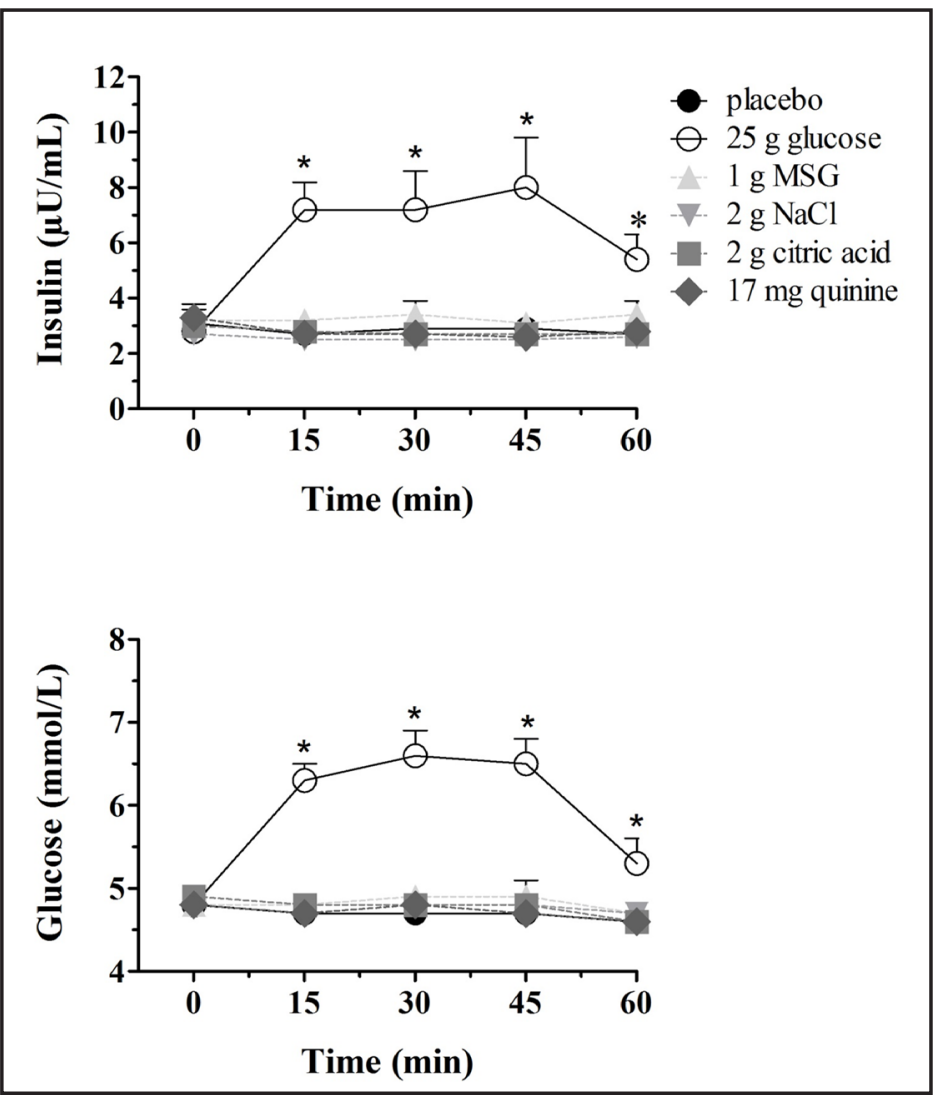

Effects of different taste substances on plasma insulin and glucose levels

Plasma insulin and glucose levels were not affected by the different taste substances (MSG, quinine, $\mathrm{NaCl}$ and citric acid) (Fig. 4). 
In contrast, glucose administration induced statistically significant elevations in plasma insulin and glucose concentrations compared to tap water and the other taste substances (AUC 0-60 min: $p<0.03$ and $p<0.007$, respectively; Fig. 4).

\section{Discussion}

The present pilot study explores 1) whether taste receptors in the upper intestinal tract can be stimulated by acute ingestion of taste stimulants bypassing the oral cavity, 2) whether these signals are conducted to the central nervous system in humans and 3) whether they influence working memory processing.

Taste is one of the most important primary oral reinforcers, driving nutrient and energy intake as well as toxin avoidance [15]. In addition, the ability to identify a distinct taste, independently of reward or aversion, and to link it by learning its appearance and location might be advantageous from an evolutionary perspective, as the source of taste can be identified in the future, where its recognition might be of use. It seems therefore plausible that taste perception and discrimination might be closely related to working memory networks and certain tastes might exhibit a modulatory effect on short-term memory. Recent studies on drosophila melanogaster showed that short-term memory was impaired when dopaminergic sweet taste receptors were blocked [16]. Our hypothesis and rationale to examine just working memory after administration of taste stimuli, is that we suggest that acute gut taste stimulation with physiological taste concentrations might impact appetitive or aversive behavior and thus influence learning tasks. It makes sense that e.g. a bitter tasting substance influences working memory in another way than sweet or umami tasting nutrients. So far, there are no human studies at all using functional brain MRI to demonstrate a link between gut taste receptors stimulation and brain activation.

In 2009 Kondoh et al. and Tsurugizawa et al. used a rat model to demonstrate that intragastric administration of glucose, MSG and $\mathrm{NaCl}$ resulted in forebrain activation on functional MRI and suggested that at least for umami sensing the vagus nerve is the major pathway transmitting this information from the gut to the forebrain as vagotomy suppressed this effect $[7,17]$. However, findings from animal studies cannot be easily transferred from one species to another, as cerebral representation of taste processing varies considerably - even among mammals. For instance rats perceive the taste of MSG as similar to that of sucrose [18]. Furthermore, in rodents, taste sensation and responsiveness to taste decreases with increasing satiety, in the sense that rats go virtually blind to sweet taste under hyperglycemia or stomach distension $[19,20]$. This stands in contrast to primate and human taste processing, where there is no habituation or modulation upon feeding state [19].

In humans, applying taste stimulants via the oral route and using functional brain MRI, insights on the cerebral localization of human taste perception could be gained: the anterior insula and adjoining frontal opercular cortex seem to represent the primary taste cortex, and caudal parts of the orbitofrontal cortex form the secondary taste cortex [21-30]. A coordinatebased meta-analysis of neuroimaging studies contrasting food tastes with tasteless solutions, confirmed insular areas as location of primary gustatory cortex. In addition, specific areas in cingulate, parahippocampal, postcentral, medio frontal and precentral gyrus, as well as caudate, claustrum, thalamus, and lentiform nucleus were found to be involved in taste processing [31]. Processing of individual taste qualities seemed to be relatively restricted to specific areas in the gustatory cortex although some anatomical overlapping exists. These findings suggest that while there is no clear chemo-topical organization, there might be at least some functional heterogeneity in this region of the human brain [28]. Recently, Dalenberg et al. investigated the functional specialization of the insula during the ingestion of the four basic tastes (sweet, salty, sour, bitter) and found that the insular cortex processes the presence of taste, its pleasantness, as well as its concentration [32]. Hoogeveen et al. measured brain activation during tasting of sweet, sour, salty and bitter taste in young and 
older adults and observed decreased brain activation in the older compared to the young volunteers in higher level brain areas of the taste pathway (especially the somatosensory areas as well as the pulvinar nucleus of the thalamus) [33]. In a pilot study, Singh et al. investigated whether there are differences in brain activation in volunteers before and after they were familiarized with the umami taste: repeated exposure to umami taste did not produce an increased response at the level of primary and secondary taste areas, but led to an additional activation of the parahippocampal gyrus (memory retrieval area) [34]. While taste perception of the oral cavity has been investigated in various trials, relatively little is known about gut taste perception and processing of this information in the human brain, which is why we chose to examine gut taste receptor stimulation.

We found that intragastric administration of quinine and MSG, representing bitter and umami taste, respectively, resulted in consistently more activated clusters than all other taste qualities. Quinine treatment recruited more clusters in the right hemispheric frontal lobe in precentral and medial frontal areas compared to saline. Besides involvement in taste reception processing, precentral areas are linked to executive functions such as the ability to differentiate among conflicting thoughts. Moreover, projection to somatosensory areas like Brodmann area (BA) 3 and 2 were found to be involved in locating objects emphasizing size and shape [35] and supplementary motoric areas (BA 6) were associated with learning complex motoric actions and visual information integration [36]. In the case of bitter taste discrimination, this could refer to the ambiguous evaluation and effect bitter tasting evokes, as it can be present to the same extent in favored edibles, pharmacologically beneficial and toxic substances. As the taste quality "bitter" is associated with conflicting information on nutrients salubriousness, it might evoke a more complex activation pattern than the "hedonic" stimulus sweet (glucose). Interestingly, while quinine treatment led exclusively to right hemispheric activation in precentral areas, MSG showed bilateral involvement of precentral areas and left hemispheric activation of postcentral and opercular areas, as well as the left precuneus and the cingulate when compared to saline treatment.

In the contrast of MSG, the cingulate gyrus, insula as well as opercular regions were recruited in the working memory task. Operculum, insula and cingulum are known regions of the primary taste cortex and activation might be related to stimulus perception. However, emotional, mnemonic, and conscious information processing regions (cingulate, precuneus, and BA 7) were also activated, thus reflecting hedonic aspects of food ingestion linked to learning [37].

We did not observe statistically significant differences in working memory processing, when we contrasted tap water and glucose. These findings are in line with recent work showing that oral water ingestion stimulates similar cortical areas as glucose [23]. This is surprising, as glucose is indicative of energy rich foods and an effect on working memory might be expected. Contrasts of citric acid did not reveal any significantly increased brain activity either. The taste quality "sour" is not strongly associated with rewarding food stimuli acids being present in fresh fruits, but also in unripe or spoilt food. A strong influence on working memory seems therefore unlikely. We found that different brain areas were involved in working memory processing upon different taste stimulation, while there was no statistically significant difference in working memory performance between different treatments.

Recently, we proposed that the acute increase in striatal connectivity to the basal ganglia network after glucose ingestion may mediate a state of satiation, which is modulated by insulin release (as the observed increase in striatal connectivity after glucose administration correlated positively with the glucose-induced insulin levels) [5]. In contrast, in the present study no increase in plasma insulin and glucose was observed in response to the different taste substances (except for glucose). These findings indicate that the altered brain activation patterns in the primary gustatory cortex and in subcortical structures after MSG and quinine ingestion are not mediated via the glucose-insulin axis and that other metabolic pathways might be responsible. 


\section{Limitations}

We would like to emphasize that our study design - intragastric taste receptor stimulation and working memory analysis in a limited number of participants - represents a pilot trial. So far, only animal studies have been carried out to show afferences from gut taste receptors via gut brain axis by use of functional brain MRI and no human studies exist to compare our findings with. We cannot rule out orthogonal effects, and we cannot distinguish direct from indirect effects. However, we are with the opinion, that our findings warrant further investigation.

From a physiological point of view, the explicit focus on gut taste receptors represents a limitation. To administer nutrients per nasogastric tube is ethologically artificial and bypasses the normal physiological route of nutrient intake, where information from the tongue and the gut are integrated. However, the reduction to intestinal taste perception might reveal a fine-grained and more specific picture for gustatory stimuli on working memory. A second limitation is the use of tap water as a placebo: Araujo et al. used aliquots of mineral water, 0.5 $\mathrm{M}$ glucose, $0.05 \mathrm{M} \mathrm{NaCl}$ and a saliva-like tasteless solution to stimulate taste receptors of the oral cavity and demonstrated that water elicits responses in similar cortical areas to those activated by prototypical tastants such as glucose and salt [23]. In the present pilot study, we ran the fMRI paradigm $15 \mathrm{~min}$. after intragastric substance administration. This was based on animal studies published by Kondoh et al. [6] and Tsurugizawa et al. [7] - the only brain fMRI study after intragastric taste stimulation we know so far - who observed slow onset of brain response upon tastant administration, with a peak for MSG at around 10 min up to $30 \mathrm{~min}$ and for glucose around 20-30 up to $60 \mathrm{~min}$. Moreover, Nakamura et al. observed different lag times for salty and sweet taste stimulation of the oral cavity, which indicates that there might even be taste-specific temporal profiles of activations in the human brain [38]. This should be taken into consideration in future studies. Another point of contention may be that the sample size in this pilot study was modest as these controlled repeated measures within-subject design studies are logistically demanding. This observation leads to a limitation in our fMRI analyses. In the fMRI group analyses we didn't use a multiple comparison correction. Although we are aware that this choice can lead to false positive results, due to the small group size we expect moderate effects of the treatments on the brain.

A further limitation in fMRI analyses is the absence of significant results for the contrast 0 -back vs. 2-back task. This may be due to the small sample size of our study that doesn't allow us to have a full overview of the effects of these treatments on the brain for each specific condition or to the actual absence of changes in the BOLD signal given by the treatments during the simple 0-back condition compared to the 2-back condition. In future studies, further investigations are therefore needed.

Therefore the following study needs to be considered as an explorative pilot study. Furthermore, we have to suggest that in future studies both sexes should be included. In conclusion, to the best of our knowledge, this is the first study to describe effects of different, intragastrically administered canonical taste qualities on working memory processing. Our findings provide preliminary evidence that umami and bitter taste are modulators of brain areas involved in working memory processing, overpowering the effects of sweet and sour reception. These findings can be interpreted from an evolutionary point of view, as it seems that the ingestion of umami, representing meaty taste, seems to have a more profound effect on learning than sweet perception. It could be argued that meat as a precious phosphate and protein source is more difficult to access than carbohydrates or sugars, and efficient learning on its retrieval could have been an evolutionary advantage. Similar conclusions could be made for "bitter" taste, which often indicates the presence of toxins. The ability to quickly remember and learn about toxin sources and to avoid them is clearly beneficial. We interpret the lack in change of activation pattern evoked by the taste quality "sour" as reflection of its ambiguous interpretation as a lot of fruits contain beneficial acids whereas spoilt foods would indicate avoidance. 


\section{SIGINAIS}

Neurosignals 2016;24:59-70

\begin{tabular}{l|l}
\hline DOI: 10.1159/000442612 & ( 2016 The Author(s). Published by S. Karger AG, Basel
\end{tabular}

Published online: July 27, 2016

www.karger.com/nsg

Meyer-Gerspach et al.: Gut Tastes Modify Brain Activity During a Working Memory Task

\section{Abbreviations}

AUC (area under the concentration-time curve); BA (Brodmann area); BMI (Body mass index); BOLD (blood-oxygen-dependent); GI (gastrointestinal); MNI (Montreal Neurological Institute); MSG (monosodium glutamate).

\section{Acknowledgements}

We'd like to thank Ernst-Wilhelm Radü, Oliver Bieri (MR facilities), Alain Thoeni and Philipp Madoerin (radiographer), as well as Luisa Baselgia, Claudia Bläsi and Sylvia Ketterer (technical assistants), and Doris Bläsi (proof reading). This research was funded by grants from the Swiss National Science Foundation (Grant No. 138 157), the Marie HeimVögtlin Foundation (Grant No. PMPDP3-145486/1) and the Stiftung zur Förderung der gastroenterologischen Forschung.

\section{Disclosure Statement}

The authors have declared that no conflict of interest exists.

\section{References}

1 Janssen S, Depoortere I: Nutrient sensing in the gut: new roads to therapeutics? Trends Endocrinol Metab 2013;24:92-100.

2 Finger TE, Kinnamon SC: Taste isn't just for taste buds anymore. F1000 Biol Rep 2011;3:20.

3 Depoortere I: Taste receptors of the gut: emerging roles in health and disease. Gut 2014;63:179-190.

4 Hofer D, Puschel B, Drenckhahn D: Taste receptor-like cells in the rat gut identified by expression of alphagustducin. Proc Natl Acad Sci U S A 1996;93:6631-6634.

5 Wolnerhanssen BK, Meyer-Gerspach AC, Schmidt A, Zimak N, Peterli R, Beglinger C, Borgwardt S: Dissociable Behavioral, Physiological and Neural Effects of Acute Glucose and Fructose Ingestion: A Pilot Study. PLoS One 2015;10:e0130280.

6 Kondoh T, Mallick HN, Torii K: Activation of the gut-brain axis by dietary glutamate and physiologic significance in energy homeostasis. Am J Clin Nutr 2009;90:832S-837S.

7 Tsurugizawa T, Uematsu A, Nakamura E, Hasumura M, Hirota M, Kondoh T, Uneyama H, Torii K: Mechanisms of neural response to gastrointestinal nutritive stimuli: the gut-brain axis. Gastroenterology 2009;137:262-273.

8 Faurion A, Cerf B, Van De Moortele PF, Lobel E, Mac Leod P, Le Bihan D: Human taste cortical areas studied with functional magnetic resonance imaging: evidence of functional lateralization related to handedness. Neurosci Lett 1999;277:189-192.

9 Smieskova R, Allen P, Simon A, Aston J, Bendfeldt K, Drewe J, Gruber K, Gschwandtner U, Klarhoefer M, Lenz C, Scheffler K, Stieglitz RD, Radue EW, McGuire P, Riecher-Rössler A, Borgwardt SJ: Different duration of atrisk mental state associated with neurofunctional abnormalities. A multimodal imaging study. Hum Brain Mapp 2012;33:2281-2294.

10 Schmidt A, Smieskova R, Simon A, Allen P, Fusar-Poli P, McGuire PK, Bendfeldt K, Aston J, Lang UE, Walter M, Radue EW, Riecher-Rössler A, Borgwardt SJ: Abnormal effective connectivity and psychopathological symptoms in the psychosis high-risk state. J Psychiatry Neurosci 2014;39:239-248.

11 Friston KJ, Ashburner JT, Kiebel SJ, Penny WD: Statistical Parametric Mapping: The Analysis of Functional Brain Images. London, 2006.

12 Ashburner J: A fast diffeomorphic image registration algorithm. Neuroimage 2007;38:95-113.

13 Schmidt A, Hammann F, Wolnerhanssen B, Meyer-Gerspach AC, Drewe J, Beglinger C, Borgwardt S: Green tea extract enhances parieto-frontal connectivity during working memory processing. Psychopharmacology (Berl) 2014. 
14 Deserno L, Sterzer P, Wustenberg T, Heinz A, Schlagenhauf F: Reduced prefrontal-parietal effective connectivity and working memory deficits in schizophrenia. J Neurosci 2012;32:12-20.

15 Rolls ET, Grabenhorst F: The orbitofrontal cortex and beyond: from affect to decision-making. Prog Neurobiol 2008;86:216-244.

16 Huetteroth W, Perisse E, Lin S, Klappenbach M, Burke C, Waddell S: Sweet taste and nutrient value subdivide rewarding dopaminergic neurons in Drosophila. Curr Biol 2015;25:751-758.

17 Kondoh T, Tsurugizawa T, Torii K: Brain functional changes in rats administered with monosodium L-glutamate in the stomach. Ann N Y Acad Sci 2009;1170:77-81.

18 Heyer BR, Taylor-Burds CC, Mitzelfelt JD, Delay ER: Monosodium glutamate and sweet taste: discrimination between the tastes of sweet stimuli and glutamate in rats. Chem Senses 2004;29:721-729.

19 Scott TR, Small DM: The role of the parabrachial nucleus in taste processing and feeding. Ann N Y Acad Sci 2009;1170:372-377.

20 Giza BK, Deems RO, Vanderweele DA, Scott TR: Pancreatic glucagon suppresses gustatory responsiveness to glucose. Am J Physiol 1993, 265:R1231-1237.

21 Barry MA, Gatenby JC, Zeiger JD, Gore JC: Hemispheric dominance of cortical activity evoked by focal electrogustatory stimuli. Chem Senses 2001;26:471-482.

22 de Araujo IE, Kringelbach ML, Rolls ET, Hobden P: Representation of umami taste in the human brain. J Neurophysiol 2003;90:313-319.

23 de Araujo IE, Kringelbach ML, Rolls ET, McGlone F: Human cortical responses to water in the mouth, and the effects of thirst. J Neurophysiol 2003;90:1865-1876.

24 de Araujo IE, Rolls ET, Kringelbach ML, McGlone F, Phillips N: Taste-olfactory convergence, and the representation of the pleasantness of flavour, in the human brain. Eur J Neurosci 2003;18:2059-2068.

25 Francis S, Rolls ET, Bowtell R, McGlone F, O'Doherty J, Browning A, Clare S, Smith E: The representation of pleasant touch in the brain and its relationship with taste and olfactory areas. Neuroreport 1999;10:453-459.

26 Iannilli E, Noennig N, Hummel T, Schoenfeld AM: Spatio-temporal correlates of taste processing in the human primary gustatory cortex. Neuroscience 2014;273:92-99.

27 Kringelbach ML, de Araujo IE, Rolls ET: Taste-related activity in the human dorsolateral prefrontal cortex. Neuroimage 2004;21:781-788.

28 Schoenfeld MA, Neuer G, Tempelmann C, Schussler K, Noesselt T, Hopf JM, Heinze HJ: Functional magnetic resonance tomography correlates of taste perception in the human primary taste cortex. Neuroscience 2004;127:347-353.

29 Small DM, Gregory MD, Mak YE, Gitelman D, Mesulam MM, Parrish T: Dissociation of neural representation of intensity and affective valuation in human gustation. Neuron 2003;39:701-711.

30 van den Bosch I, Dalenberg JR, Renken R, van Langeveld AW, Smeets PA, Griffioen-Roose S, Ter Horst GJ, de Graaf C, Boesveldt S: To like or not to like: neural substrates of subjective flavor preferences. Behav Brain Res 2014;269:128-137.

31 Huerta CI, Sarkar PR, Duong TQ, Laird AR, Fox PT: Neural bases of food perception: coordinate-based metaanalyses of neuroimaging studies in multiple modalities. Obesity (Silver Spring) 2014;22:1439-1446.

32 Dalenberg JR, Hoogeveen HR, Renken RJ, Langers DR, Ter Horst GJ: Functional specialization of the male insula during taste perception. Neuroimage 2015.119:210-220.

33 Hoogeveen HR, Dalenberg JR, Renken RJ, Ter Horst GJ, Lorist MM: Neural processing of basic tastes in healthy young and older adults - an fMRI study. Neuroimage 2015;119:1-12.

34 Singh PB, Hummel T, Gerber JC, Landis BN, Iannilli E: Cerebral processing of umami: A pilot study on the effects of familiarity. Brain Res 2015;1614:67-74.

35 Stoeckel MC, Weder B, Binkofski F, Buccino G, Shah NJ, Seitz RJ: A fronto-parietal circuit for tactile object discrimination: an event-related fMRI study. Neuroimage 2003;19:1103-1114.

36 Nachev P, Kennard C, Husain M: Functional role of the supplementary and pre-supplementary motor areas. Nat Rev Neurosci 2008;9:856-869.

37 Hayden BY, Platt ML: Neurons in anterior cingulate cortex multiplex information about reward and action. J Neurosci 2010;30:3339-3346.

38 Nakamura Y, Goto TK, Tokumori K, Yoshiura T, Kobayashi K, Honda H, Ninomiya Y, Yoshiura K: The temporal change in the cortical activations due to salty and sweet tastes in humans: fMRI and time-intensity sensory evaluation. Neuroreport 2012;23:400-404. 